This item was submitted to Loughborough's Research Repository by the author.

Items in Figshare are protected by copyright, with all rights reserved, unless otherwise indicated.

\title{
A framework for material flow assessment in manufacturing systems
}

PLEASE CITE THE PUBLISHED VERSION

http://dx.doi.org/10.1080/21681015.2014.1000403

\section{PUBLISHER}

Taylor \& Francis $@$ Chinese Institute of Industrial Engineers

\section{VERSION}

AM (Accepted Manuscript)

\section{PUBLISHER STATEMENT}

This work is made available according to the conditions of the Creative Commons Attribution-NonCommercialNoDerivatives 4.0 International (CC BY-NC-ND 4.0) licence. Full details of this licence are available at: https://creativecommons.org/licenses/by-nc-nd/4.0/

\section{LICENCE}

CC BY-NC-ND 4.0

\section{REPOSITORY RECORD}

Gould, Oliver, and James Colwill. 2019. "A Framework for Material Flow Assessment in Manufacturing Systems”. figshare. https://hdl.handle.net/2134/20722. 


\title{
A FRAMEWORK FOR MATERIAL FLOW ASSESSMENT IN MANUFACTURING SYSTEMS
}

\author{
Oliver Gould*, James Colwill \\ Wolfson School of Mechanical and Manufacturing Engineering, \\ Loughborough University, Loughborough, LE11 3TU, United Kingdom
}

\begin{abstract}
Improving material efficiency is widely accepted as one of the key challenges facing manufacturers in the future. Increasing material consumption is having detrimental impacts on the environment as a result of their extraction, processing and disposal. It is clear that radical improvements in material efficiency are required to avoid further environmental damage and sustain the manufacturing sector. Current resource management approaches are predominantly used to improve material consumption solely in economic terms. Meanwhile, environmental assessment methodologies can determine sources of significant environmental impact related to a product; however, a methodology to effectively assess material efficiency in production systems is currently not available. This paper highlights the benefits of material flow modelling within manufacturing systems to support advances in increased material efficiency, proposing a framework for 'material flow assessment in manufacturing' that promotes greater understanding of material flow and flexibility to explore innovative options for improvement.
\end{abstract}

Keywords: material efficiency, modelling; framework, MFAM; manufacturing, production, system.

\footnotetext{
* Corresponding email: O.J.Gould@lboro.ac.uk
} 


\section{INTRODUCTION}

Resource Efficient Manufacturing (REM) is a process that has traditionally been driven by market competition as manufacturers seek to reduce their production costs to maximise profits and increase sales [1]. This primarily focuses on reducing labour, materials and energy costs, whilst increasing production efficiencies and output. Whilst these previous drivers for REM have been largely economic more recently, as REM has been adopted as a sustainability strategy, the drivers have expanded to include resource conservation, 'doing more with less' clearly aligning with the goals of sustainable manufacturing and environmental improvement (less waste, fewer resources, etc.). Sustainability led REM will often place a greater focus on the use of materials, water and energy, while labour and capital costs are usually secondary considerations. Therefore, whilst many of the past strategies, methods and tools for REM, such as 'Lean Manufacturing' and 'Process Optimization' are still relevant and useful, they are potentially inadequate for identifying the additional improvements that will be required to meet the sustainability goals and manufacturing challenges of the future.

In companies where resource efficiency has been driven largely by cost reduction targets, managers will have naturally focused on those resources which have the greatest financial impact on the business. For most manufacturers these would be primarily materials, labour, overheads and distribution. At some point, in order for the company to remain competitive, they would need to consider each of these resources. However, there have been certain periods where there has been a particular focus on, or a trend towards the optimisation of one particular resource. Such as offshoring to reduce labour costs or as a response to external pressures such as the energy spikes in the 70's, material shortages, wage inflation, or more recently environmental levies, legislation and waste management. However, with materials often accounting for around $50 \%$ of the production cost (as illustrated in Figure 1, [2]) it is reasonable to assume that this particular resource would remain a high priority for most manufacturers.

Scarcity of resources has been identified by the European Factories of the Future Research Association as one of the seven critical trends for manufacturers [3]. The degree of scarcity for a particular resource may be both regionally and seasonally specific, such as with water, or largely 'global demand' driven such as is the case with energy. This view as to which materials are critical or scarce is largely a subjective and dynamic one depending on the industry, country and demand at a given time [4]. In 2010, a list of 14 critical materials were drawn up by the EU, and referred to as the EU-14 [5]. However, this list is as much geopolitically influenced, as it is reflective of a particular materials availability and consumption. One of the problems with classifying materials as a separate resource is that material availability can be intrinsically linked with the other resources such as energy and water.

\section{MATERIAL EFFICIENCY STRATEGIES (MES)}

Material efficiency has been described as the provision of a product or service with less material production [6]. This has clear environmental benefits if the amount of the same material previously required for the manufacture of a product is reduced as the associated impacts for that material will also be proportionally reduced. Whereas if this reduction requires a change of material, the associated benefits are less clear and a more detailed assessment of the environmental impacts of the two different materials is needed. Material efficiency strategies (MES) can be grouped into two types, those which change what is manufactured (design changes) and those change only how it is manufactured (process changes), however changes to the product may also require process changes (Figure 2). A number of methods and techniques can be employed during the design stage that can improve the material efficiency of a product, such as material elimination, minimisation or substitution, however these will often be visible to the consumer and will require cross departmental approval as well as technical and market trials. During production, material efficiency may be improved without altering the final product by reducing yield losses: the amount of material lost during manufacturing processes, for example, through subtractive processing or through quality control failures [6]. This may be achieved by examining and optimising individual processes; by substituting processes for those with improved yield; or by altering the material flow through an entire production system. Production optimisation may involve implementing a range of strategies or changes: from altering production planning or scheduling, to altering factory layout or developing more flexible or reconfigurable manufacturing systems. One of the key advantage s of process only changes are that they can be achieved within the responsibility remit of a single department (manufacturing) and should not require market or product trials.

These two approaches may be considered in isolation; however, combining design and process changes has the potential to deliver the greatest gains. However, as previously highlighted, implementing design changes to an existing product can be a complex and costly task, the design of a product is therefore best optimised at the outset rather than retrospectively, therefore the initial focus of this research has 
been on REM that does not require changes to the product specification.

Furthermore, although the aforementioned broad definition of material efficiency (product service with less material production) is important to bear in mind, more specific and detailed definitions of material efficiency may be necessary to uncover complexities and fully understand the trade-offs and benefits involved with implementing MES. Further work is needed to develop ways to provide material efficiency definitions that are most appropriate for specific applications.

Analysis of the flow of materials through manufacturing systems may be used to improve material efficiency, in particular for identifying opportunities to improve yield in production. The following sections briefly describe a number of existing methodologies and strategies that are relevant to material efficiency in production.

\section{REVIEW OF MATERIAL FLOW MANAGEMENT METHODOLOGIES}

The well-established Material Requirements Planning (MRP) methodology [2], gives a measure of the raw materials/components input demand which a manufacturer has per product. The purpose of this planning is to calculate the demand for materials and components in line with production scheduling. MRP therefore requires information in the form of the bill of materials (BOM), supply chain lead times for obtaining materials and production schedules. This can help to optimise the flow of materials into a production system and minimise inventory levels. However, a BOM will only record the information of an input at its stored item level, to identify individual materials used this data will need to be combined with additional information such as the raw material's product specification, and what additional factors have been used for allocation of waste. Furthermore MRP does not provide any description of the internal dynamics of how materials flow through a system, thus is not able to highlight critical areas of inefficiency and opportunities for improvement within the system. Empirical models evaluate inputs and outputs but provide no consideration of internal dynamics instead adopting a 'black box' approach. They are built on quantitative relationships rather than process understanding and generally have low data requirements.

Material Flow Analysis (MFA) is a methodology for investigating the metabolism of anthropogenic and environmental systems in space and time [3]. MFA is based on the mass balance principle: that matter is conserved in any system, thus input is equal to output mass. The literature describes various applications of this methodology, predominantly at the scale of national and geographic systems [4-7]. However, MFA may be appropriate for quantifying the mass flow of materials at smaller scales, such as manufacturing systems, to locate and examine inputs, partitioning, outputs and important sources of waste materials in the factory. MFA of a manufacturing system may be used to highlight the areas of inefficient material use and losses to waste. This MFA methodology defines three types of processes relevant to manufacturing: transformation, transport and storage of materials [3]. However material flow assessment specifically for manufacturing may also include inspection and delay processes. A manufacturing material flow model can be represented using input-output network diagrams, or simple Sankey diagrams, which can visualise the relative flow efficiency in systems [8]. As MFA is not specifically tailored for the analysis of manufacturing systems, it has a number of shortfalls when used in this application. MFA is best suited to the analysis of large systems that tend to remain static over time, giving visibility to the quantities of materials and their fate within the system. This static view of a system gives limited scope for modifying the model, which is a major disadvantage, as manufacturing systems are in general readily modifiable, as compared to economic systems. This is particularly problematic for the future of manufacturing, as systems are generally moving towards being more flexible, dynamic and reconfigurable in their nature.

Strategies contained within lean manufacturing [9], such as Just in Time (JIT) [10], and Total Quality Management (TQM) [11] can have an influence on material efficiency. In material flow terms, lean strategies aim to reduce unnecessary stock (minimising material residence time in a system); reduce waste material production and improve quality of outputs (minimising quality control waste).

Although lean manufacturing activities have been correlated with reduced quantities of waste and emissions [12], this does not necessarily indicate improved material efficiency in every case. Lean strategies tend to view efficiency solely in economic terms and do not take into account other ways in which material efficiency may be defined. For example, implementing lean strategies may achieve an overall waste reduction in economic terms but have an increased net environmental impact; efforts to reduce one type of material waste may interact with and result in increasing waste output of a different material type that has a significantly greater environmental impact, but is not as costly.

To improve the characterisation of waste material flows in manufacturing, Hicks et al. [13] developed a methodology for modelling material flow, broadly characterising process waste according to the waste hierarchy (Lansink's ladder). This information is plotted against the cumulative value of the materials 
embedded in each unit product during each process and aims to identify potential value streams associated with process wastes. Employing this method may have an impact on material efficiency related to improving waste management. However, decisions based on broad characterisation of waste according to the waste hierarchy correlated to economic value may not result in reduced environmental impact.

Although the broad principle of using the waste hierarchy to drive decisions to reduce environmental impact is conceptually sound in many cases, it does not allow decisions to be made based on empirical evidence. Thus, significant factors may be hidden which may be misleading as to the environmental (or cost) benefit of alternative waste management options. Producing waste that is higher in the waste hierarchy does not necessarily reduce the environmental impact in every case [14][15]. A more systematic approach as used in Life Cycle Assessment (LCA, ISO14040) is to quantify the outputs from a process (emissions) in terms of its quantity, characterisation and allocation to a particular impact type. However, LCA models a product functional unit rather than a production system and so is not suitable for dynamic modelling of material flow, or for providing options for material efficiency improvement in the manufacturing context.

\section{FOCUS OF RESEARCH}

The previous section clearly highlights that there is a lack of support for determining the consequences of implementing material efficiency strategies in the production stage. Furthermore, methods, which may be used to examine material flow and associated environmental impact, are not sufficiently focussed on production to support manufacturer's decision making.

As materials flow through manufacturing processes (transport, storage and transformation), quantitative changes occur which are measureable and accounted for using methods such as MFA, using mass balance approach. Current methods are focussed on analysing quantitative aspects of material use; however, as materials flow through a system, they are often subject to qualitative changes which have significant impact on material flow and properties, which may define environmental impact. Currently there are no methods that effectively model material flow both in quantitative and qualitative terms. We propose that material flow must be assessed in these terms to comprehend the full complexity of material flow and identify opportunities for ME improvement. In particular, the qualitative changes, which occur during transformation processes, must be fully understood.

A methodology that will allow both dynamic material flow modelling and characterisation of waste generated at different parts of a system through mod- elling material transformations (in qualitative and quantitative terms), will support engineers in the optimisation of a production system to maximise material efficiency. Currently models and methodologies that allow materials to be modelled do not characterise materials or waste flow in sufficient detail, while methodologies to assess impacts of material use (qualitative and quantitative) do not allow for effective flow modelling in manufacturing systems.

The improved characterisation of materials and therefore waste will therefore help to identify sources of significant impacts that must be eliminated preferentially using the best combination of resource efficiency strategies in order to have the most significant reduction of environmental impact. Thus, the methodology will improve on lean manufacturing, which aims to eliminate all waste without discrimination, regardless of positive or negative environmental benefit. It is reasonable to assume that, in most manufacturing processes, there are always inefficiencies and so a certain amount of waste will always be unavoidable. Therefore by modelling the material flow and wastes produced in terms of their environmental impacts will help ensure that the overall impact of all the waste produced is minimised. A framework that uses material flow modelling to assess material efficiency in terms of both economic and environmental impact criteria is therefore proposed, that provides options for improvement that are transparent in terms of cost and environmental benefit. It is worth clarifying that the framework and methodology proposed is intended firstly for the assessment of existing production systems and secondly is complimentary to existing manufacturing management and production design methods rather than as an alternative or replacement to them.

\section{QUALITATIVE MATERIAL FLOW DURING MANUFACTURING}

Quantitative material flow is based on the concept of mass balance, which is derived from the principle of mass conservation, i.e. that matter cannot be destroyed or created, although it may be converted or rearranged in space. In distinction to quantitative changes, which must always be balanced in terms of overall inputs and outputs, the conversion of matter (transformation) can result in the creation or destruction of qualitative material properties. For example, the material input and output mass quantities for a rubber vulcanising process must always balance; however, certain qualitative properties of the input rubber are destroyed upon the crosslinking of its constituent polymer chains. It can be seen that key area for incorporating qualitative data is at manufacturing 
process level. The three types of material processes, as defined by MFA are: transformation, transport and storage. We propose that the most important qualitative information and the greatest sources of qualitative variation in materials are associated with transformation processes.

There is a large range of manufacturing processes, with a large variety of associated transformations that may affect different materials in different ways, both quantitatively and qualitatively. A key qualitative aspect of material transformations is the dependencies, which may exist between consecutive transformation processes; one process may not be able to proceed before another has finished. For example, filling a container before the container itself is produced. In this case, the manufacturing transformation, of raw material into physical container, must take place before the transformation of an empty to filled container can occur.

A further key qualitative aspect of certain transformations is their reversibility. Referring again to the rubber vulcanisation process, this transformation can currently be described as irreversible. Reversibility is not an absolute term however, as all transformation processes may theoretically be reversible, provided the knowledge, technology and resources exist to carry out the process. Hence, reversibility may be described as a relative term, referring to the practical methods by which it may be carried out. For example, a coating transformation process (Figure 3) involving the combination of two materials may be described as reversible via a range of methods, such as the dissolution (a transformation process which alters the physical state of the coating), or mechanical scraping to remove the coating material. Qualitatively, the reversal of a coating transformation may only be applicable to one of the two materials, i.e. the coated material is returned to its original form, however, the coating material is further transformed by dissolution or scraping and is not returned to its original form.

The reversibility of transformations is particularly important when considering the characteristics and management of waste materials; the reversibility can dictate the possible options for waste flow. A fully reversible transformation may allow wasted material to be reused, following reversal of the transformation. An irreversible transformation may result in waste material that is not reusable or recyclable, with limited potential for recovery of constituent reusable materials.

It is not currently possible to model qualitative changes in material flow. To address this, a framework for material flow assessment in manufacturing has been developed, which incorporates both quantitative and qualitative information. This will form a comprehensive material flow accounting and assessment method to inform mechanisms which may be used to generate and assess alternative strategies for improving material efficiency in manufacturing.

The following section details a framework which provides a systematic approach to conducting material flow assessment in manufacturing (MFAM).

\section{FRAMEWORK FOR MATERIAL FLOW ASSESSMENT IN MANUFACTURING}

\subsection{Framework specification}

The MFAM framework is to be used as a basis for investigation of the material efficiency in the manufacture of products. The assessment of material efficiency is based on the objectives of using less material processing to produce a unit product, to improve production yield and to reduce the environmental impact of material processing, as described in the previous sections. The framework approach has the purpose of giving structure to the investigation of complex systems, with the aim of uncovering innovative opportunities for improvement. The framework must facilitate the analysis of material flow through static and deterministic modelling to identify locations of inefficient material flow, defined and measured with appropriate parameters. In addition, material flow simulation (stochastic modelling) of alternative production strategies should be facilitated as a method of determining if material efficiency can be improved.

Many of the terms defined in MFA (substance, good, material, process, activity, flow and flux etc.) are used with equivalent definition in this framework. However, the MFAM framework is designed to be distinct from MFA, to focus clearly on the assessment of manufacturing systems.

\subsection{Framework Phases}

The material flow assessment framework consists of five distinct phases as shown in Figure 4. These phases are largely interdependent, with phases 1-4 following a largely sequential but potentially iterative progression. The fifth phase meanwhile 'interpretation' runs in parallel with the other phases as an iterative and reflective mechanism interpreting the results and applying suitable decision making process.

\subsubsection{Phase one: production system scope}

MFAM begins with an explicit statement as to the aims and objectives of the study. The scope of the study must then be specified. This involves the defi- 
nition of the system boundaries, products and processes, and begins with the specification of the physical processes, equipment, lines, factories etc. to be included in the study. In turn the products that are manufactured wholly or partially by all or part of this system can then be identified and selected or omitted for inclusion in the study. This includes defining the production system (e.g. factory) that is being examined and the products being manufactured within this system. The function and requirements of the system are clearly defined to provide a means of comparison between the performance of the existing system and any options for improvement of material flow efficiency. Thus a subject-specific system boundary is created.

In defining the scope of the MFAM the following items should be considered and clearly described:

- $\quad$ The functions of the production system

o What products are required

o What quantities are required

- The production system to be studied

o Spatial boundary

o Temporal boundary

o Product boundary

- Material flow efficiency assessment criteria and method

o What aspect or aspects of material flow are the focus

- Information requirements

o What is known or measured

o What do we need to know or measure

- $\quad$ Specific assumptions or barriers

o Standard processes

o Standard operating procedures

- $\quad$ GMP or other accredited procedures

The first task in defining the manufacturing system is to state its function and purpose: what the required outputs of the system are. Both the manufactured products and the quantity of products per unit time need to be defined. Where there is flexibility in system function (e.g. where output is variable), this needs to be described.

The manufacturing system must be defined in order to define the overall system boundary. The manufacturing system is defined in terms of its spatial and temporal system boundaries. This is similar to defining the system boundary in MFA methodology.

A system boundary should include processes and events which can be directly controlled and monitored. Manufacturing systems contain various measurement and control systems that alter the flow of materials over time. These points of control should be defined in terms of their locations, influence and a description of the method of control. The control system may be automated, utilising feedback mechanisms, or personnel may be responsible for (manual) process con- trol. The automated and manual control systems, or combinations must be identified.

Manufacturing processes include transformation, transport and storage processes. Process subdivision should be performed to the extent of identifying the smallest, fundamental process units that can be directly controlled. For example, if a controllable process contains a number of individual sub-processes that are not directly controllable, the boundary should be set at the overall controllable process.

Each fundamental process is connected physically with other processes, forming the various pathways for the flow of resources. Processes may be physically connected. The system boundary should describe the spatial arrangement of each manufacturing element and the physical connectivity between processes (input and output connections) including cyclic systems. This includes detailing each of the manufacturing processes used, the process grouping, zoning or manufacturing cells that are present. An illustration of how primary and secondary system boundaries can be set for a multi-line production facility is shown in Figure 5.

Definition of the temporal system boundary includes stating how the production system operates over time. This includes information as to production scheduling and the method of operation with respect to time for each element, e.g. batch, semi-continuous or continuous processing. The temporal system boundary should be set for each manufacturing element at a level which reflects the frequency of data acquisition, i.e. every minute, every hour, daily, weekly, or according to shift or batch frequency.

The product boundary is important to define; depending on the system, multiple products or semi-complete products may be the output. Furthermore, the inputs may be semi-complete products. This must be defined to properly understand the function of the system and how much control can be expected over the range of products that are fully or partially completed within the system.

Products made entirely within the system boundary should be categorised as primary products, whereas secondary products are those which are partially processed within and partially processed outside of the system boundary.

For materials which are inputted as part of sub-components that are pre-assembled outside of the factory, it is necessary to define if they fall into the scope for detailed investigation in this framework. For instance, the materials within the sub-components may be within scope if the investigator can have an influence on their manufacture. If not, it is important to determine the sub-components contribution to the material mass of the product and to assess if this is significant.

Information relating to the products manufactured should include the design and statement of the 
product function and service. Product groups or families contributing to the same activity (e.g. detergent, to clean) comprising specific products which vary by a particular parameter (e.g. scent and label) should be described in this detail. For example, a product group may vary by the colour of a certain component; the product activity and service do not vary, but the materials vary to provide alternative colours (with the assumption that this example of aesthetic variation does not alter product service). Variations in a product group that alter the product service should be described, based on the interrelation between product and service variation. Where product group variation impacts the manufacturing system, for example through the requirement for additional processes to provide the product variation, these details should be noted in the definition of the manufacturing system boundary.

Products may be grouped for assessment where variations are not considered significant. If a range of related products are made on a line within the system boundary, then the relative time spent making each variant should be quantified. The categorisation of products as primary and secondary products coupled with assessing the products that are predominant within a system will allow prioritised improvement of the most significant product outputs.

Once the manufacturing system boundary has been defined, it is then important to outline how material flow efficiency is to be assessed in the system. The aspects of material efficiency which are the focus should be clearly stated, to assist in the selection of criteria for assessment.

The information requirements for the study should then be stated, outlining what general information is required for the study and what information is available for the study. The data acquisition points should be determined, including location of process monitoring equipment or sensors and the frequency of data acquisition. The quality of data availability should be addressed in terms of its precision, completeness and uncertainty. The data requirements must be set out and compared to the data availability.

Any specific assumptions or requirements must be stated. Standardised processes and operating procedures may present barriers for system alteration, depending on the manufacturer and system. Similarly, any aspects of manufacturing practice that are required for certain manufacturing accreditations (e.g. Good Manufacturing Practice, GMP) must be stated and adhered to.

\subsubsection{Phase two: material flow inventory}

Inventory analysis involves attributing data and information to elements contained within the system boundary defined in the previous phase. This is achieved by defining the inputs, the processes that act upon them and the resulting outputs. This phase creates a material flow model based on mass balance through the system, detailing both quantitative and qualitative flow within individual transformation, transport and storage processes. The physical connectivity of process inputs and outputs are assembled to complete the system model, giving the overall product and waste outputs in qualitative and quantitative terms.

A complete inventory is required including all the substances and raw materials that are required to manufacture the products within the defined system. Material consumption during manufacturing includes both materials embedded in the final product and the materials which are not embedded in the final product but are required for production. The former can be described as embedded materials (EM) and the latter are described as auxiliary materials (AM). An example of EM is the steel embedded in the chassis of a motor vehicle and AM would be the cutting fluid and cutting tool used in producing the chassis. EM information may be collected by referring to the bill of materials (BOM) for a product. A material may be both embedded in a product but required in the process in excess quantities. An AM may or may not be a consumable material, that is, it may be continually recycled, or consumed during the process (e.g. cutting tool erosion).

In order to create a quantitative material flow model it is necessary to assign the quantity of each material per unit product. This provides an initial description of material requirements, which will serve as a starting point for the definition of flows through the system. This includes quantities of EM and AM; the latter should be estimated by determining the rate of consumption divided by the rate of production (in unit products per unit time). Quantitative EM information may again be collected from BOM for the product.

Material Flow Assessment involves investigating material flow according to a range of qualitative and quantitative measures. To facilitate this, it is necessary to include detailed semi-quantitative and qualitative material information in addition to qualitative mass flow data. The fundamental descriptors of each material should be included such as the substances which it is comprised of, its primary functional parameters (physical, mechanical, aesthetic, etc.), hazard information and storage information. Other inherent descriptive factors relating to waste management and environmental impact should be stated such as waste management options, material recyclability and options for recycling, or if a material is from recycled feedstock. This inventory process serves as a database of key qualitative and quantitative parameters for the materials flowing through the system. Thus each material in the inventory can be characterised using a range of factors that are important to material efficiency. This characterisation should in part be based on self-assessment. Different manufacturers or facto- 
ries have different access to services, resources and other supply chains; for material information contained within an assessment model to be meaningful, it must be relevant to the system that is being modelled.

It is important to assign economic value to materials as they flow though processes. Economic value of materials may decrease or increase as they are transformed in manufacturing processes. For example, per equivalent mass, the value of raw steel increases as it is cast into billet steel. Subtractive processing of billet steel (e.g. cutting) produces steel off-cuts (scrap), which have decreased value. Conversely, the required steel piece may have increased value once combined with other materials to produce a high value sub-component. A description or estimation of material accessibility and predicted future accessibility may assist in prioritising targets for material efficiency improvement.

Hazard information for a material as it flows through processes is important to describe, to assess the implications of a manufacturing process on the hazards posed by a material. For example, elemental Mercury is more hazardous (to health) in the vapour phase than in liquid phase and this increases further when combined with a methyl group to produce organic methyl-mercury. Environmental hazards should also be described along with health hazards.

Taking a life cycle view of materials requires that their recyclability be assessed and detailed. Examples of materials that cannot be recycled are vulcanised rubber and composites. Conversely, many metals such as steel can be recycled relatively easily with no loss of material properties. Recycled material inputs should be described. Recycled steel has same properties as billet steel; however, other recycled materials may have reduced or enhanced material properties. Recycled materials may have differences in texture, appearance or other aesthetic properties.

Material footprint is a description of the resources required to produce a certain mass of the raw material. For example, in general, the extraction of target metals from ores requires significant amounts of ore to be processed. The footprint of the ore may be extended to include the other resource inputs required for metal extraction and refining, as well as the transport of finished metals.

Certain materials have been used in manufacturing for many decades and generally there are numerous established options for processing these. On the other hand, novel or high tech materials generally have less well established options for processing, which may limit the options for alternative processing routes. A manufacturer may also have limited access to alternative processing options, due to budgetary, space, resource or legislative limitations. Thus, a description of a material's manufacturability will illustrate the practical feasibility of alternative production scenarios.
Once the input materials for the entire system have been characterised and defined, it is then necessary to define the function of process units which act upon these materials. The inputs and outputs to each process within the previously defined system must be determined. This information must be combined with information on qualitative and quantitative changes to materials during each transport, transformation and storage process. Thus, a quantitative and qualitative material flow model is constructed that conveys a complete description of the fate of materials within the system. Including information on the changes to material characteristics in quantitative and qualitative terms (e.g. partitioning of materials, reversibility of transformations, respectively) will produce a comprehensive material flow model for interrogation in the assessment phase.

\subsubsection{Phase three: material flow assessment}

This includes steps to examine the material flow model according to various performance measures related to material efficiency. By applying different performance assessment criteria, the material flow can be assessed based on a range of metrics that appraise the qualitative and quantitative mass flow through a system. Simple mass flow metrics such as production yield can be used alongside increasingly descriptive metrics, from proportion of waste sent to landfill, to hazardous waste production etc.

\subsubsection{Phase four: improvement scenario modelling}

The selection and testing of potential improvement strategies takes place in the penultimate phase. At its most basic, a paper-based study can be investigated and improvement scenarios can be designed and assessed alongside the original system model, to determine the impact and define potential benefits to material efficiency according to a range of performance metrics. Alternatively, in silico simulation modelling of strategy implementation may be used to increase the scope of data handling and potential options; information collected and organised by the framework may be entered into software tools designed to simulate the implementation of improvement strategies. A separate, modified model of the system will be created (modifying system and also the material flow inventory). This will create a new model with altered qualitative and quantitative material flow, which can then be assessed and compared according to the material flow efficiency assessment phase criteria.

\subsubsection{Phase 5: interpretation}

Interpretation is a continuous and iterative process that takes place during the study and is also the final phase of material flow assessment, in which the 
findings from the material flow inventory and assessment are considered alongside results from improvement strategy simulations and assessments. In this phase it is determined how best to improve material efficiency in the manufacture of a product. The findings of this interpretation take the form of detailed assessment, breaking down the material flow efficiency and material processing impacts, to inform decision making towards improvements.

\section{EXAMPLE MATERIAL FLOW ASSESSMENT}

The following example assessment aims to describe the application of some of the concepts and methods described in the MFAM framework.

\subsection{Production system scope}

A system is defined with the function of producing bottled beverage, in a single product variety. The bottling system boundary is set to include the following processes: bottle moulding, filling, capping and labelling. The current production sequence is bottle moulding, filling, capping and finally labelling. The purpose of material flow assessment is to examine the waste produced in the system in terms of quality control rejects. A measurement of the number of rejects produced at each process is carried out every 100 products, giving average percentage efficiency per process.

\subsection{Material flow inventory}

Respectively, per unit product, bottle moulding adds polyethylene terephthalate (25 g), filling adds beverage (330 g), capping adds high density polyethylene (4 g) and foamed polyethylene (1 g), and the labelling process adds polyvinyl chloride (1.5 g) and adhesive (0.5 g).

Bottle moulding involves the conversion of liquid material into solid material (EM) and is defined as reversible, bottles can be remoulded. Filling combines the solid bottle material with the liquid beverage contents (EM). This is a reversible process but the contents must be sent to waste whereas the bottle can be reused. Capping combines the solid bottle and cap materials (EM), sealing the bottle. This process is reversible; however, the cap sealing mechanism is damaged during reversal and the cap is wasted, the bottle may be reused. Labelling involves combining the bottle material with the label material (EM) using an adhesive (EM). This process is reversible; however a detergent (AM) is required to remove the adhesive and the label must be wasted.
The process transformations dictate the process precedence constraints: that filling and labelling must follow moulding and that capping must follow filling. The rejection rate of each process is $1 \%$.

\subsection{Material flow assessment}

The purpose of this material flow assessment is to determine the quality control reject waste produced. The rejection rate of each process is equal (99\% efficient). Thus for every 100 products, 4 products are rejected overall and 96 products are completed. The current production sequence is bottle moulding, filling, capping and finally labelling, hence the overall cumulative yield loss is $1102 \mathrm{~g}$.

\subsection{Improvement scenario modelling}

Unlike other assessment methodologies, this framework includes an analysis phase which identifies a range of alternative configurations feasible within the limitations imposed by the programmer. For this example the process sequence configuration is examined to provide improvements, by altering the production system but not altering the processes within the system or product itself. Based on the precedence constraints for each process, there are two other feasible sequence permutations: moulding, filling, labelling and capping; or moulding, labelling, filling and capping. The former permutation has $1099 \mathrm{~g}$ of yield loss and the latter produces $771 \mathrm{~g}$. Thus, by reconfiguring the process sequence, a significant difference in overall yield loss in terms of mass can be the result.

\subsection{Interpretation and Validation}

The alternative scenarios generated in phase four of the framework are largely theoretical and based on the information available from the current system. Changing a process sequence may introduce additional problems which were not present in the original system. In our bottle example, labelling the bottles before filling reduces the number of rejected filled bottles from a misplaced label. However, any spillage that occurs during the filling process could now stain the label, a problem that would not occur in the original configuration. It is therefore necessary that each alternative scenario produced by the model is reviewed by one or more of the experienced factory managers/engineers and the assumptions tested. Where weaknesses in the model are identified, these can be corrected and then phase four repeated. As with other assessment methodologies, this phase is intended to be an iterative process. 


\section{CONCLUSIONS}

This paper describes a framework for material flow assessment within the manufacturing facility. This uses a combined quantitative and qualitative approach to model and assess material flow efficiency. The framework primarily supports the investigation of material flow in a manufacturing system to uncover innovative opportunities for improvement that might not normally be uncovered by current approaches. A conceptual basis for the development of both paper based and computationally based system modelling is given, facilitating dynamic material flow modelling, to identify opportunities for improved material efficiency within the production system boundaries without impacting the design of products contained within. Thus, the approach enables the development of descriptive models as well as providing a foundation for prescriptive modelling, to suggest options to consider for improvement.

The material flow assessment framework described here will provide the basis for development of a material flow assessment support tools which will facilitate:

- Optimisation of process sequencing to maximise the environmental and economic material efficiency of the overall production system.

- Individual process optimisation, assessed in terms of the overall system impact.

- $\quad$ Process substitution, which may or may not include material substitution (product design change), depending on the product-system boundary.

- Optimisation of operational control, process and production scheduling

- Investigation of more wide ranging system alterations, e.g. implementation of flexible lines or reconfigurable elements.

Decision making support will be provided through the generation of optimum solutions described using a range of parameters; providing economic and environmental evidence to build a business case for change. This novel and progressive approach to material flow modelling will allow for innovative improvements in manufacturing material efficiency.

Further work involves the development of decision support tools, to facilitate the modelling of large production systems. The volume and complexity of the data acquired from these systems is likely to be most efficiently assessed by computerised tools which implement the MFAM framework implementation by enabling model construction and the use of dynamic algorithms for automated generation of improvement options.

\section{ACKNOWLEDGEMENTS}

This work was funded by the Engineering and Physical Sciences Research Council as part of the Centre for Innovative Manufacturing in Industrial Sustainability.

\section{REFERENCES}

1. Allwood JM, Ashby MF, Gutowski TG, Worrell E. Material efficiency: A white paper. Resour Conserv Recycl 2011;55:362-81.

2. Plossl GW, Orlicky J. Orlicky’s Material Requirements Planning. 2nd ed. McGraw-Hill Professional; 1994.

3. Brunner PH, Rechberger H. Practical Handbook of Material Flow Analysis. Int J Life Cycle Assess 2004;9:337-8.

4. Finnveden G, Moberg Å. Environmental systems analysis tools - an overview. J Clean Prod 2005;13:1165-73.

5. Kleijn R. Adding It All Up The Sense and Non-Sense of Bulk-MFA. J Ind Ecol 2000;4:7-8.

6. Geyer R, Davis J, Ley J, He J, Clift R, Kwan A, et al. Time-dependent material flow analysis of iron and steel in the UK: Part 1: Production and consumption trends 1970-2000. Resour Conserv Recycl 2007;51:101-17.

7. Davis J, Geyer R, Ley J, He J, Clift R, Kwan A, et al. Time-dependent material flow analysis of iron and steel in the UK: Part 2. Scrap generation and recycling. Resour Conserv Recycl 2007;51:11840.

8. Schmidt M. The Sankey Diagram in Energy and Material Flow Management. J Ind Ecol 2008;12:82-94.

9. Womack JP, Jones DT, Roos D. The Machine that Changed the World: The Story of Lean Production. New York, USA: HarperCollins Publishers; 1990.

10. Monden Y. Toyota Production System: An Integrated Approach to Just-In-Time, 4th Edition. CRC Press; 2011.

11. Shah R, Ward PT. Lean manufacturing: context, practice bundles, and performance. J Oper Manag 2003;21:129-49.

12. King AA, Lenox MJ. Lean and Green? an Empirical Examination of the Relationship Between Lean Production and Environmental Performance. Prod Oper Manag 2001;10:244-56.

13. Hicks C, Heidrich O, McGovern T, Donnelly T. A functional model of supply chains and waste. Int J Prod Econ 2004;89:165-74.

14. Price JL, Joseph JB. Demand management - a basis for waste policy: a critical review of the 
applicability of the waste hierarchy in terms of achieving sustainable waste management. Sustain Dev 2000;8:96-105.

15. Schmidt JH, Holm P, Merrild A, Christensen P. Life cycle assessment of the waste hierarchy - A Danish case study on waste paper. Waste Manag 2007;27:1519-30.

\section{ABOUT THE AUTHORS}

Oliver Gould is the lead researcher on the Resource Efficient Manufacturing Grand Challenge Project in the Engineering and Physical Sciences Research Council Centre for Innovative Manufacturing in Industrial Sustainability. He received his $\mathrm{PhD}$ in Biosciences from The University of Nottingham, his research interests are resource efficiency and material flow in manufacturing, sustainable design of products and production systems.

James Colwill is a lecturer in sustainable manufacturing at Loughborough University and is the principle investigator for the Resource Efficient Manufacturing Grand Challenge Project in the Engineering and Physical Sciences Research Council Centre for Innovative Manufacturing in Industrial Sustainability. He received his PhD in Manufacturing and Mechanical Engineering from Loughborough University and his research interests include resource modelling, mapping, and conservation through recycling and remanufacturing, life cycle assessment, sustainable design and manufacturing strategies.

(Received September 2013; revised November 2013; accepted December 2013) 